\title{
WOLF-RAYET GALAXIES
}

\author{
WILLIAM D. VACCA and PETER S. CONTI \\ Joint Institute for Laboratory Astrophysics \\ University of Colorado, Boulder, CO 80309-0440, USA
}

Wolf-Rayet (W-R) Galaxies are a subset of starburst galaxies (usıally, blue compact dwarfs) in whose integrated spectra a broad He II $\lambda 4686$ emission feature has been detected (e.g., Osterbrock and Cohen 1982; Kunth and Joubert 1985). This line is a prominent emission feature in the spectra of Galactic WN stars. The presence of $10^{2}$ to $10^{5} \mathrm{~W}-\mathrm{R}$ stars in these galaxies has been inferred from a comparison of the line fluxes in the integrated galaxy spectra with those jn the spectra of Galactic WN stars (e.g., Kunth and Schild 1986; Armus, Heckman, and Miley 1988).

In an effort to perform a systematic study of the properties of W-R galaxies, we have obtained moderate resolution $(\sim 3.5 \AA)$ spectra of 10 known $W-R$ galaxies and 4 comparison starburst galaxies using the 2D-Frutti detector on the 4-m telescope at CTIO. The spectra cover the wavelength range $\sim 3120$ to $\sim 7000 \AA$. We report on these observations and a preliminary analysis of the spectra of these galaxies.

In all $10 \mathrm{~W}-\mathrm{R}$ galaxies, the He II $\lambda 4686$ feature is resolved, with a typical FWHM of $\sim 14 \AA$. In the spectra of $3 \mathrm{~W}-\mathrm{R}$ galaxies a broad (FWHM $\sim 20 \AA$ ) $\mathrm{N}$ III $\lambda 4638$ emission feature is also detected. Both of these lines are characteristic features of the spectra of late WN or Of/WN stars. The absence of C III and C IV emission lines in the spectra suggest that the WC/WN number ratio in these galaxies is very small, similar to that found in the low metal abundance environments such as the LMC and SMC. The blue continua and strong nebular emission lines (e.g., the Balmer series, [O II], [O III], and [Fe III]) indicate that the W-R. galaxies have higher excitation, and therefore, many more hot stars, than the comparison starburst galaxies. The relatively weak or absent Balmer absorption lines and Balmer jumps in the spectra of $W-R$ galaxies indicate that the hot stars must be the dominant contributors to the overall galactic spectra. These galaxies may be greatly scaledup examples of less energetic extragalactic "giant" II II regions. We interpret W-R galaxies as examples of the youngest phase of the starburst phenomenon, in which massive stars are still being born or star formation has only recently ended (less than a few $\times 10^{6}$ years ago).

\section{REFERENCES}

Armus, L., Heckman, T. M., and Miley, G. K. 1988, Ap. J. (Letters), 326, L45.

Kunth, D., and Joubert, M. 1985, Astr. Ap., 142, 411.

Kunth, D., and Schild, H. 1986, Astr. Ap., 169, 71.

Osterbrock, D. E., and Cohen, R. D. 1982, Ap. J., 261, 64. 